\title{
Continuum EVN and MERLIN observations of Ultra Luminous Infrared Galaxies
}

\author{
A.G. Polatidis ${ }^{1}$ and J.E. Conway ${ }^{2}$
}

1 Max Planck Institut für Radioastronomie, Bonn, Germany
2 Onsala Space Observatory, Sweden

Abstract. Radio imaging of ULIR galaxies is ideal to explore the connection between the starburst and the AGN phenomenon since it is unaffected by dust obscuration, and provides the required high angular resolution to distinguish between an AGN and starburst emission. We have made combined $18 \mathrm{~cm}$ radio continuum, EVN and MERLIN observations of 13 ULIRGs that have the parsec and deci-parsec scale resolution necessary to distinguish between an AGN and supernovae remnants at the centres of these galaxies, and assess the contribution of each to the total energy distribution. Images of four galaxies are presented here.

\section{Introduction}

To date the best studied sample at high resolution of ULIRG galaxies is the IRAS Bright Galaxy sample (hereafter the BGS sample). Condon et al. (1990 1991) observed over 40 compact sources with the VLA from 1.6 to $8 \mathrm{GHz}$. Lonsdale et al. (1993) L93) made snapshot VLBI images of 31 sources from Condon et al. (1991); of these 21 were detected in VLBI baselines greater than $10 \mathrm{M} \lambda$, prompting Londsale et al. to suggest that an AGN was responsible for the VLBI structure. However, Smith et al. (1998a), in full track observations of Arp 220 showed that the VLBI structure consists of radio supernovae or supernovae remnants. Following this, Smith et al. (1998b) modeled , the L93 data fitting clustered luminous compact radio supernovae, showing that for most sources the VLBI structure could be explained by starburst activity while for only a few sources the presence of an AGN was invoked to account for the parsec scale structure.

We are engaged in a systematic survey of the complete sample of the 19 northern $(\delta>0)$ compact ULIRGs in the BGS sample which showed evidence for detections $>1 \mathrm{mJy}$ on at least one baseline longer than $10 \mathrm{M} \lambda$. Of these 19 compact ULIRGs six (IIIZw 35, Mrk 231, Mrk 273, Arp 220, NGC 7469 and Mrk 331) were already well observed at the same resolution, hence we were left with 13 objects to observe.

We have observed the sources with a combined MERLIN and EVN array at $18 \mathrm{~cm}$ so that we can separate the diffuse emission at MERLIN scales which can be due to thermal emission combined with synchrotron emission from electrons from supernovae diffused into the ISM, from the compact radio emission which can be due to an AGN or to synchrotron emission associated with single or clustered radio supernovae or supernovae remnants (SNRs).

\section{Observations}

Initially the five brighter sources were observed with the combined MERLIN and EVN array, in February 2002. The remaining 8 sources were observed in May 2003. In this paper we present preliminary results from the February 2002 observations.

The sources were observed with six telescopes of the EVN (Torun, Medicina, Onsala, Effelsberg, Westerbork, and the Jodrell Bank Lovell and Mark 2 telescopes alternating during the observations) with a sampling rate of $256 \mathrm{Mbits} / \mathrm{s}$ over a $32 \mathrm{MHz}$ bandwidth. The MERLIN observations had a $15 \mathrm{GHz}$ bandwidth in overlapping frequency range. Due to the relative weakness of the sources, the observations were made in phasereferencing mode with a cycle of $7 \mathrm{~min}$ on the source and 3 minutes on the nearby phase calibrator.

The observations were partially successful. Of particular importance was the failure of the Cambridge telescope which would have provided the common baseline (Lovell Cambridge) to facilitate the cross-calibration of the two arrays, and the subsequent reduction of the MERLIN resolution to $\sim 250$ mas. Furthermore the loss of the Lovell telescope for $40 \%$ of the run, although it was replaced by the Jodrell Bank Mark 2 antenna, resulted in reduction of sensitivity of the array. Consequently the array consisted of the 6 MERLIN telescopes and $6 \mathrm{EVN}$ antennae at the most for each source.

\section{Results}

The EVN data were correlated at the JIVE data processor and were then processed with the AIPS software suite. Initial amplitude calibration and fringe fitting was done with the EVN pipeline and was refined later.

\subsection{UGC 05101}

UGC 05101 at VLA scales shows a $\sim 1.57 \times 1.14 \operatorname{arcsec}(1110 \times$ $810 \mathrm{pc}$, at a distance $\mathrm{d}=158 \mathrm{Mpc}$ ) disk-like emission elongated roughly east-west, dominated by a central bright component (seen clearly in Condon et al's (1991) 8.4 GHz image). At the MERLIN resolution of 270 mas (Fig. 16 we detect a similar structure measuring $933 \times 533 \mathrm{pc}$ (roughly $1.3 \times 0.75 \mathrm{arcsec}$ ). The flux density in the MERLIN image is consistent with that of Condon et al. (1990) and Thean et al. (2001). 

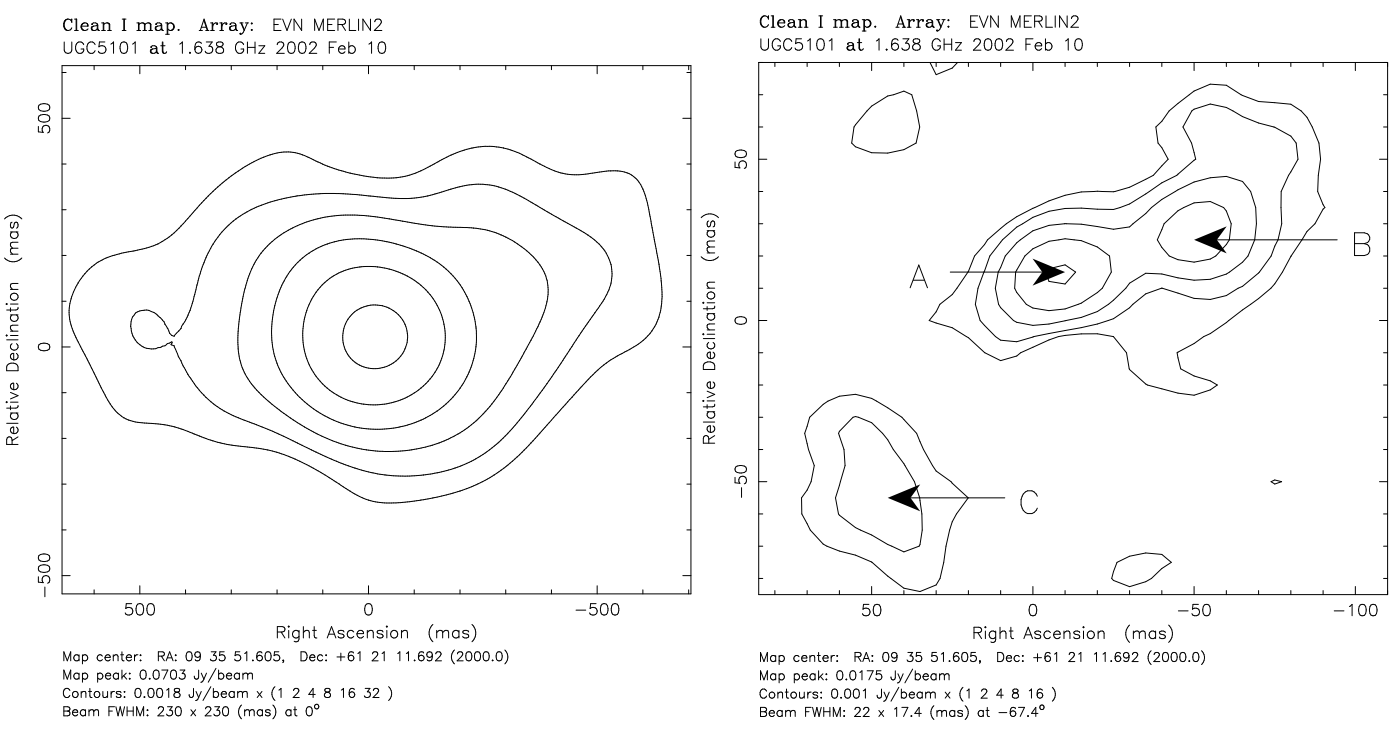

Fig. 1. Left: The MERLIN image of UGC 05101 with a restoring beam of 230 mas. The peak is $70.3 \mathrm{mJy} / \mathrm{bm}$ and the contours are drawn at 1,2,4,8,16,32 x $1.8 \mathrm{mJy} / \mathrm{bm}$. Right: The EVN image of UGC 05101 restored with a beam of $22 \times 17.4$ mas. The peak is $17.5 \mathrm{mJy} / \mathrm{bm}$ and the contours are drawn at $1,2,4,8,16 \mathrm{x} 1 \mathrm{mJy} / \mathrm{bm}$.

In the highest resolution ( $15 \mathrm{pc}$ ) EVN image $\sim 53 \%$ of the flux density is recovered in an apparently S-shaped source extending roughly $128 \times 140$ parsec. Three major components (A-C), relatively compact (5-13 pc) are embedded in weak extended radio emission, visible in the lower resolution images that are not presented here. Component A is closer to the peak brightness of the MERLIN image. The brightness temperatures of components $\mathrm{A}$ and $\mathrm{B}$ are $\sim 10^{7} \mathrm{~K}$ while that of the more resolved component $\mathrm{C}$ is $710^{6} \mathrm{~K}$, while their luminosities range from $510^{39}$ to $10^{40} \mathrm{erg} / \mathrm{s}$.

Lonsdale et al. (2003) with higher resolution $5 \mathrm{GHz}$ global VLBI observations find a consistent nuclear structure, while they resolve A and B in two compact subcomponents each.

These compact components are $\sim 50$ times more luminous than the brightest radio supernovae (RSN) in Arp220. They are also resolved and their sizes are too large for single RSN. It appears that the nuclear structure of UGC 05101 can not be associated with RSN but is rather caused by an obscured AGN. This is consistent with Imanishi et al.'s (2003) detection of Fe $\mathrm{K} \alpha$ line emission in $\mathrm{X}$-rays, though the $\mathrm{X}$-ray spectrum was measured with an aperture of 8 arcsec $(5.6 \mathrm{kpc})$ which covers the whole radio source and hence it is not easy to orrelate the position of the X-ray source and the radio emission. Multifrequency observations are needed to further explore this possibility.

\subsection{NGC 6286}

NGC 6286 is an edge-on spiral galaxy (at a distance $\mathrm{d}=73 \mathrm{Mpc}$ ) in an interacting pair with NGC 6285 ( 1'.5, $30 \mathrm{kpc})$ classified as a LINER. Condon et al. (1990) interpret the $1.6 \mathrm{GHz}$ VLA image, showing a 20" (6.8 kpc) resolved source elongated along the optical axis as starburst induced.

Our MERLIN image (Fig. 2) recovers $37 \mathrm{mJy}(\sim 25 \%$ of the VLA flux density) and consists of two-components sepa- rated by 450 mas $(150 \mathrm{pc})$ at the same orientation as the VLA image. At the 25 mas resolution of the EVN image, the brighter MERLIN component is resolved in two components (A, B) separated by $18.4 \mathrm{pc}$, with the brightest B located at the northeast. 200 parsec northeast of B, we detect the resolved out component $\mathrm{C}$, which is the weaker component of the double in the MERLIN image.

All three components in NGC6286 are resolved with sizes between 4 and 10 parsecs, brightness temperatures ranging from $1.0210^{6} \mathrm{~K}(\mathrm{C})$ to $8.910^{6} \mathrm{~K}(\mathrm{~B})$ and luminosities from $10^{37}$ to $10^{39} \mathrm{erg} / \mathrm{s}$, hence a few times higher than the RSN in Arp 220.

The fact that they are resolved argues against single RSN. It is plausible that one of the compact components may be due to a clump of supernovae. It is also possible that the brightest component are due to AGN activity.

\subsection{Mrk 266}

Mrk 266 is a system of interacting galaxies (at a distance $\mathrm{d}=113$ $\mathrm{Mpc})$ showing an interaction region in the middle and tidal tails. Mazzarella et al. (1988) classify the northern as a LINER and the southern galaxy as a Seyfert 2 type. VLA and MERLIN images show at least three co-linear components of which the outer are associated with the two interacting galaxies and the central may be a region of synchrotron emission stimulated by the collision of the two galaxies with the interaction region. With increasing resolution the two southern components become resolved while the northern (which is the target of our EVN observations) remains compact.

In our MERLIN image (Fig. 2), the northern component has a flux density of $21 \mathrm{mJy}$, consistent with the image of Thean et al. (2001) and a spectral index $\alpha_{1.6-8 G H z}=0.93$. The higher resolution EVN observations (beam=21mas, $107 \mathrm{pc}$ ) show a relatively compact $(2 \times 2 \mathrm{pc})$ component $(3.4 \mathrm{mJy})$ and arc-like 

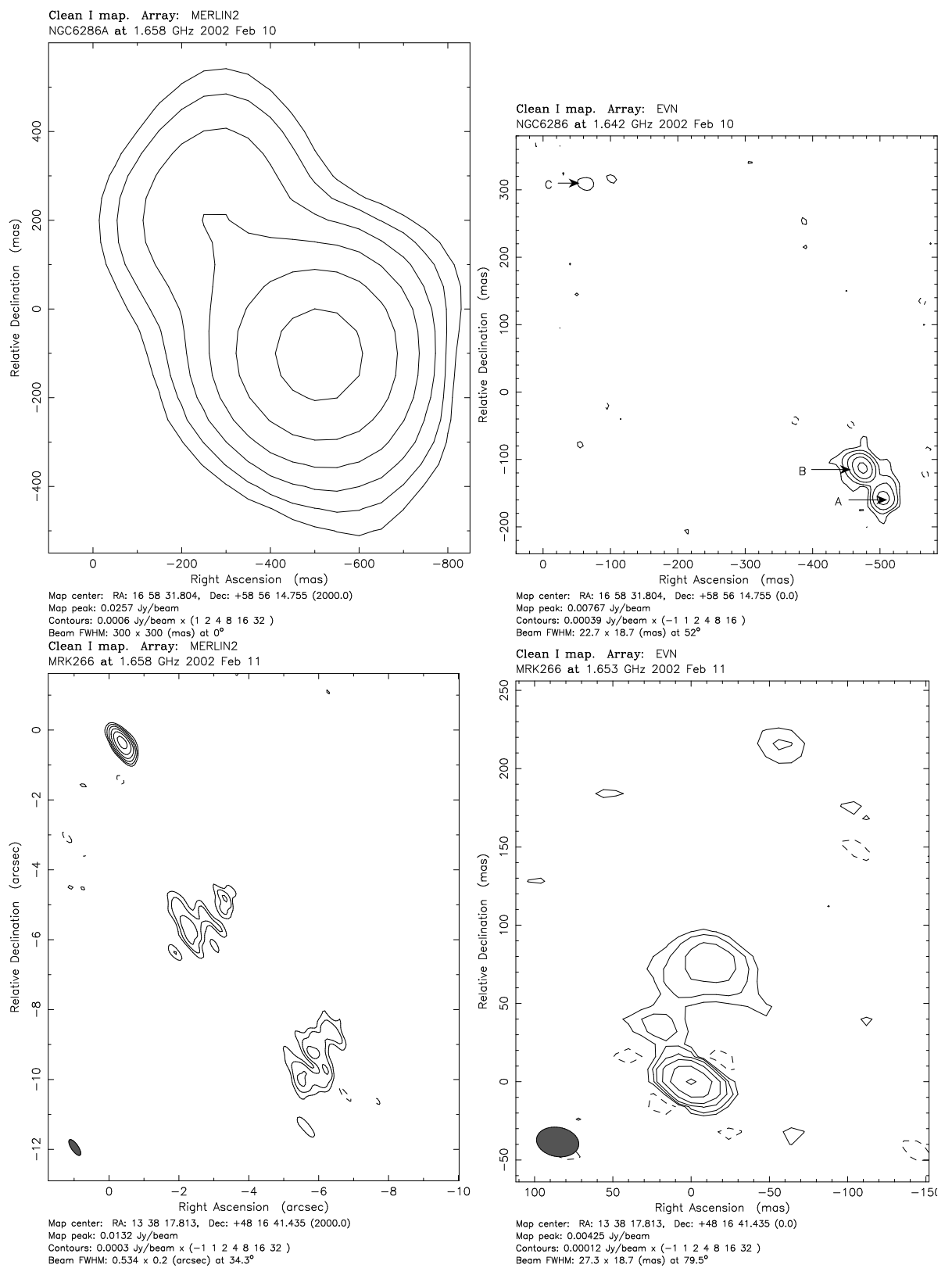

Fig. 2. Top Left: The MERLIN image of NGC6286 with a restoring beam of 300 mas. The peak is $25.7 \mathrm{mJy} / \mathrm{bm}$ and the contours are drawn at 1,2,4,8,16,32 x $0.6 \mathrm{mJy} / \mathrm{bm}$. Top Right: The EVN image of NGC6286 restored with a beam of $22.7 \times 18.77 \mathrm{mas}$. The peak is $7.6 \mathrm{mJy} / \mathrm{bm}$ and the contours are drawn at 1,2,4,8,16 x $0.39 \mathrm{mJy} / \mathrm{bm}$. Bottom Left: The MERLIN image of Mrk 266 with a restoring beam of $534 \times 200$ mas. The peak is $13.2 \mathrm{mJy} / \mathrm{bm}$ and the contours are drawn at 1,2,4,8,16,32 x $0.3 \mathrm{mJy} / \mathrm{bm}$. Bottom right: The EVN image of Mrk 266 restored with a beam of $27 \times 18$ mas. The peak is $4.2 \mathrm{mJy} / \mathrm{bm}$ and the contours are drawn at $1,2,4,8,16 \times 0.12 \mathrm{mJy} / \mathrm{bm}$.

emission which culminates in a more extended $(18 \times 14 \mathrm{pc})$ weaker $(1.7 \mathrm{mJy})$ subcomponent $\sim 41 \mathrm{pc}$, while a third weak component is visible $\sim 100 \mathrm{pc}$ further north. Their brightness temperature range from $2.310^{7} \mathrm{~K}$ to $3.310^{5} \mathrm{~K}$ and their luminosity from $410^{38}$ to $8.210^{38} \mathrm{erg} / \mathrm{s}$.

Combining our EVN image with archival MERLIN 5GHz data, we find that the brightest, compact component has a steep spectrum of $\alpha_{5-1.6 \mathrm{GHz}}=0.681$, while weaker and more extended component has a rising spectrum of $\alpha_{5-1.6 \mathrm{GHz}}=-0.023$.

\subsection{NGC 2623}

NGC 2623 is a LINER galaxy (at a distance $d=74.138 \mathrm{Mpc}$ ) whose optical appearance suggests a late state of merging. A single bright component is seen at the centre of the galaxy at near infrared images (the nucleus is heavily obscured at the $\mathrm{V}$ band). Lipari et al (2004) find evidence for two starburst events, a circumnuclear burst with an age of 1 Gyr and a compact nuclear starburst with an age of $10 \mathrm{Myr}$.

Condon et al. (1991) detected a well resolved structure with the VLA at $8 \mathrm{GHz}$ indicating a starburst origin for the radio 

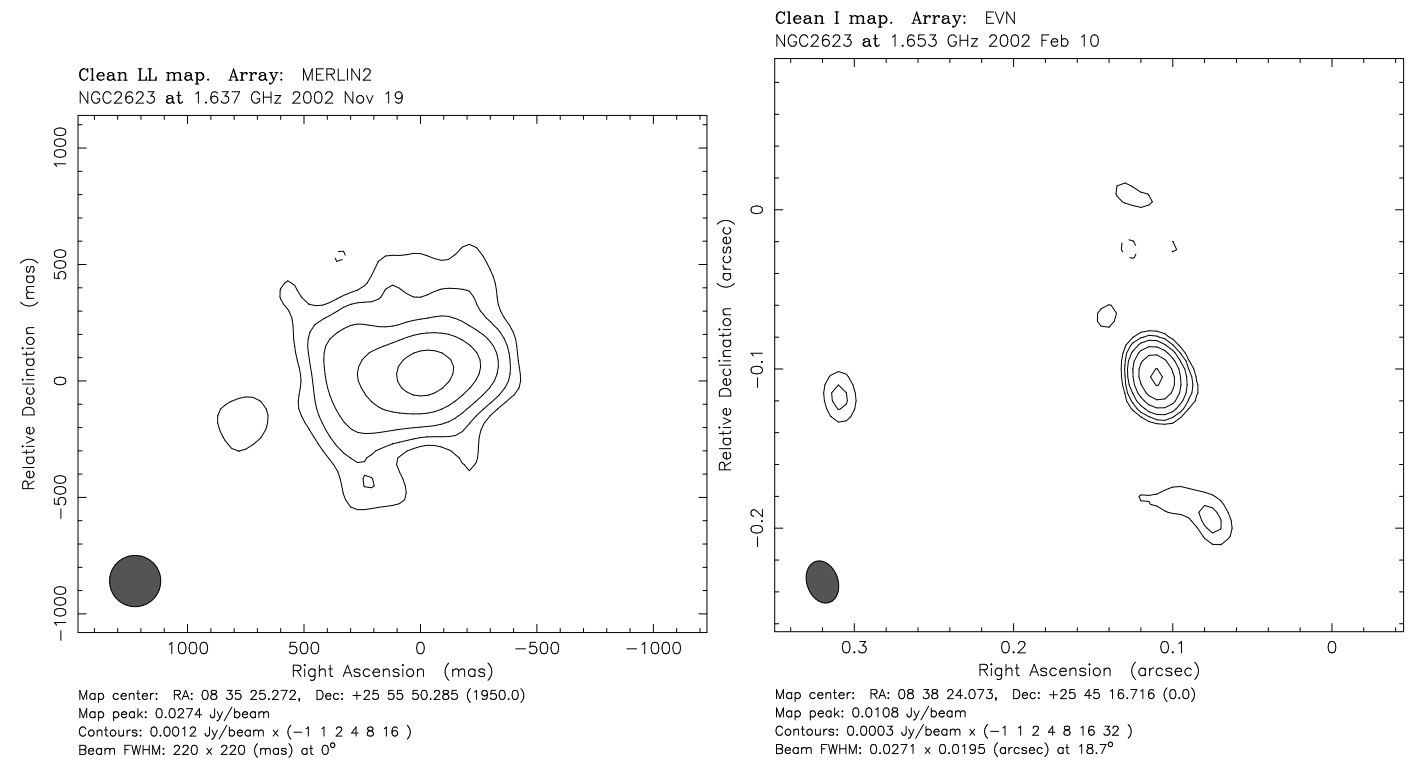

Fig. 3. Left: The MERLIN image of NGC 2623 with a restoring beam of 220 mas. The peak is $27.4 \mathrm{mJy} / \mathrm{bm}$ and the contours are drawn at 1,2,4,8,16,32 x $1.2 \mathrm{mJy} / \mathrm{bm}$. Right: The EVN image of NGC 2623 restored with a beam of 27x19 mas. The peak is 10.8 $\mathrm{mJy} / \mathrm{bm}$ and the contours are drawn at $1,2,4,8,16 \times 3 \mathrm{mJy} / \mathrm{bm}$.

emission, while Lonsdale et al (1993) found evidence for a high brightness compact component embedded in complicated radio emission.

Our MERLIN image (Fig. 3) shows a slightly asymmetric structure, elongated in a roughly east-west direction, extending for $\sim 350 \mathrm{pc}$. The EVN image shows at least two $(\sim 6 \mathrm{x} 6 \mathrm{pc})$ components, separated by $70 \mathrm{pc}$, with the brightest having luminosity of $1.0310^{39} \mathrm{erg} / \mathrm{s}$ and a brightness temperature of 3.9 $10^{6} \mathrm{~K}$ while the weaker has a luminosity of $8.110^{37} \mathrm{erg} / \mathrm{s}$ and a brightness temperature of $3.910^{5} \mathrm{~K}$. At least one other weak and extended component, is detected $\sim 96 \mathrm{pc}$ to the south of the brightest component with $\mathrm{T}_{B}=9.610^{4} \mathrm{~K}$ and a luminosity of $5.110^{37} \mathrm{erg} / \mathrm{s}$.

Given that the shape of the hard X-ray spectrum can only be explained by a Compton-thick, cold, reflection dominated AGN (Maiolino et al, 2003) it is plausible that the brightest component in the EVN image is the location of a weak AGN, while for the other two components a starburst origin is plausible.

\section{Conclusions}

In the four ULIRGs discussed in this paper, $50 \%$ to $70 \%$ of the total flux density is resolved out at parsec scale resolution. The parsec scale structure consists of multiple components with sizes of a few to tens of parsecs, brightness temperatures ranging from $10^{5}$ to $10^{7} \mathrm{~K}$ and luminosities from $10^{37}$ to $10^{40} \mathrm{erg} / \mathrm{s}$ (UGC 05101). All components are too large to be single radio supernovae and apart from UGC 05101, their luminosities are a few thousand times brighter than Cas A but similar to the supernova 1989J. It is therefore possible that at least some of these components are clustered young radio supernovae (RSN) or supernovae remnants.

It appears that at least some of the high brightness temperature parsec scale emission is due to an AGN, in particular in
UGC 05101 whose components are much more luminous than 1989J or the supernovae in Arp 220. This plausibility needs to be explored with further multi-frequency observations as well as X-ray observations.

The picture that seems to be emerging is that in most ULIRGs, starbursts provide most of the energy, however a detectable AGN is present in a fraction of those and in those cases a significant minority of the energy (10\%-40\%) can come from AGN accretion. Recent X-ray observations (e.g. Ptak et al (2003) add further support to this picture since strong AGNlike X-ray sources are found with CHANDRA and Beppo-SAX in a significant fraction of ULIRGs.

Acknowledgements. The European VLBI Network is a joint facility of European, Chinese, South African and other radio astronomy institutes funded by their national research councils.

\section{References}

Condon, J. J., Helou, G., Sanders, D. B., \& Soifer, B. T., 1990, ApJS, 73,359

Condon J.J., Huang Z.-P., Yin Q.F. \& Thuan T.X., 1991, ApJ 378, 65

Imanishi M, Terashima Y., Anabuki N., Nakagawa T., 2003, ApJ, 596, L167

Lipari S., Mediavilla E.,, Diaz R.J., Garcia-Lorenzo B., Acosta-Pulido J., Aguer M. P., Terlevich R., 2004, MNRAS, 348, 369

Lonsdale C.J., Lonsdale C.J., \& Smith, H. E. 1992, ApJ 391, 629

Lonsdale C.J., Smith H. \& Lonsdale C.J., 1993, ApJ 405, L9

Lonsdale C.J., Lonsdale, C.J., Smith H.E., Diamond P.D., 2003, ApJ, 592, 804

Mazzarella J.M., Gaume R.A., Aller H.D., Hughes P.A., 1988, ApJ, 333,168

Maiolino R.,Comastri A., Gilli R., Nagar N. M., Bianchi S., Bker T., Colbert E., Krabbe A., Marconi A., Matt G., Salvati M.,2003, MNRAS, 344, L59

Ptak A., Heckman T., Levinson T., Weaver K., Strickland D., 2003, ApJ, 592, 782 
Smith H.E., Lonsdale C.J., Lonsdale C.J. \& Diamond P.J., 1998a, ApJ 493, L17

Smith H.E., Lonsdale C.J. \& Lonsdale C.J., 1998b, ApJ 492, 137

Thean A.H.C, Gillibrand T.I., Pedlar A. , Kukula, M.J., 2001, 327, 369 